\title{
Overview of Techniques for THz QCL phase-locking
}

\author{
$\underline{\text { A. Khudchenko }}{ }^{1,2}$, D.G.Pavelev ${ }^{3}$, V.L Vaks ${ }^{4}$, A.M. Baryshev ${ }^{1,5}$ \\ ${ }^{1}$ Kapteyn Astronomical Institute/NOVA, University of Groningen, Groningen, Netherlands, a.khudchenko@ sron.nl \\ ${ }^{2}$ Kotel'nikov Institute of Radio Engineering and Electronics RAS, Moscow, Russia \\ ${ }^{3}$ Lobachevsky State University, Nizhny Novgorod, Russia \\ ${ }^{4}$ Institute for Physics of Microstructures RAS, Nizhny Novgorod, Russia \\ ${ }^{5}$ Astro Space Center, Lebedev Physical Institute of Russian Academy of Science, Moscow, Russia
}

Since the first demonstration of a THz Quantum Cascade Laser (QCL) in 2002 [1] it was rapidly improved towards practical applications, for example THz imaging [2] and molecular spectroscopy [3] with high resolution.

At the moment, QCL is one of the most attractive continuous wave $(\mathrm{CW})$ sources in the frequency range of 3-6 $\mathrm{THz}$ for heterodyne spectroscopy. In the terahertz astronomy QCLs are used as LO sources in heterodyne receivers for projects SOFIA [4] and GUSTO [5] and are considered for space observatories such as Millimetron [6], LOCUS [7] and OST [8]. Frequency stability of LO is absolutely crucial for a heterodyne instrument because it influences the quality of scientific data delivered by the receiver. The most reliable option to stabilize the LO frequency is phase-locking. Phase-locking of a THz QCL has been demonstrated by various groups using various methods. Here we make an overview of these techniques.

The first frequency stabilization was demonstrated by Betz et al. in 2005 [9] by frequency-locking (and partial phase-locking) of $3 \mathrm{THz} 1 \mathrm{~mW}$ QCL to a far-infrared (FIR) gas laser using GaAs Schottky mixer. A while later, in 2006 Baryshev et al. [10] showed phase-locking of two-mode QCL, using an inter-mode beat signal generated on hot-electron bolometer mixer (HEB). Both these experiments were important demonstration of QCL stabilization, though they were difficulties for practical applications.

Afterwards, in 2009 Rabanus et al. [11] and Khosropanach et al. [12] showed independently stable phase-locking for single-tone QCL, in [11] it was used $1.5 \mathrm{THz} 0.3 \mathrm{~mW}$ laser and in [12] $-2.7 \mathrm{THz}$ $0.38 \mathrm{~mW}$ one. The key moment is that both groups used HEB mixer to generate the beat signal for phase locking loop (PLL) system. The reference RF signal in [11] was generated by a high power multiplying chain able to pump an HEB mixer, while in [12] was used a frequency comb generated by superlattice diode mixer [13] pumped by $182 \mathrm{GHz}$ microwave source. A little later, in 2010 Consolino et al. [14] realized similar approach for phase-locking of $2.5 \mathrm{THz} 1 \mathrm{~mW}$ QCL, using HEB mixer, though the reference RF reference was a COMB signal generated by the Cherenkov effect in a lithium niobate waveguide effected by Ti:sapphire femtosecond laser. These three phase-locking approaches [11][12][14] can be combined in one group show schematically on fig.1, utilizing HEB mixer, which is known to be the most sensitive mixer from 1.5 to $6 \mathrm{THz}$. Nether the less, presence of HEB an in the locking scheme means arranging a separate $4 \mathrm{~K}$ cryostat. It is not an issue for lab experiments, but it makes serious complications for on-bard application of a locking system.

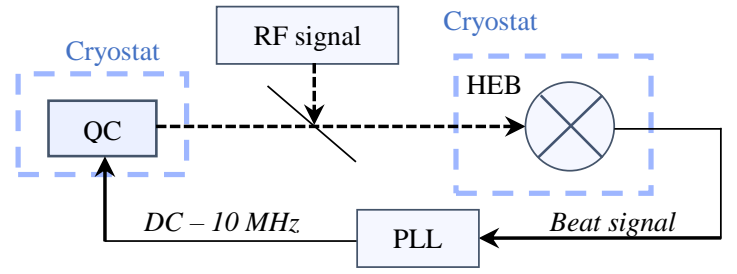

Fig. 1. Simplified block-diagram of QCL phase-locking described in papers [11], [12] and [14]. In all the cases the HEB mixer is used to provide a beat signal between the QLC and a reference signal. The RF signals in all the approaches are generated in different way.

In 2010 Barbiere et al. [15] demonstrated another approach of QCL phase-locking. In this experiment, a mode-locked femtosecond laser and a QCL radiation $(2.7 \mathrm{THz}$, power of $25 \mathrm{~mW})$ were applied to electrooptic detection system. This photo-mixing system generated a mixing product between a QCL frequency and the $\mathrm{n}_{\text {th }}$ harmonic of repetition rate of femtosecond laser of $90 \mathrm{MHz}$. The photocurrent beat signal frequency was $30 \mathrm{MHz}$. The electro-optic detection system is based on ZnTe crystal modulating amplitude of femtosecond pulses with the QCL frequency and on high speed (bandwidth of $300 \mathrm{MHz}$ ) silicon photodiodes. Similar technique was used a year later in 2011 by Ravaro et al. [16] to phase-lock $10 \mathrm{~mW}$ QCL radiating at $2.5 \mathrm{THz}$ (see fig. 2).

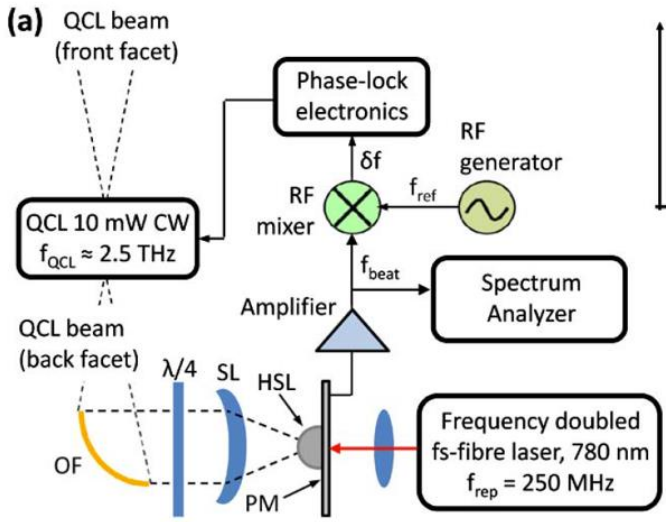

Fig. 2. Block-diagram of QCL phase-locking taken form [16]. THz field from the QCL is mixed with the photocarrier $\mathrm{THz}$ comb induced in the PM by the fs pulse train generated in fiber laser with repetition rate of $250 \mathrm{MHz}$.

The main new element relative to [15] is a GaAs photomixer. A big advantage of scheme in [15] and [16] is that only a room-temperature-operated elements are used. A fiber laser technology and the semiconductor 
photomixer are much more light and compact than superconducting bolometer mixers.

Another way of QCL phase-locking has been described in 2013 by Hayton et al. [17]. The authors phase locked a $3.4 \mathrm{THz} 1 \mathrm{~mW}$ distributed feedback (DFB) laser to the 18th harmonic of a $190.7 \mathrm{GHz}$ reference source using a room temperature GaAs/AlAs superlattice diode. Next year Khudchenko et al. [18] employed the same technique for $4.7 \mathrm{THz} 0.25 \mathrm{~mW}$ QCL. The key element of this scheme (see fig. 3) is the harmonic mixer receiving QCL signal and mixing it with $\mathrm{n}_{\text {th }}$ harmonic of a reference microwave signal of about $10 \mathrm{~mW}$ power at frequency around $200 \mathrm{GHz}$. The product is used by PLL system to control phase and frequency of QCL. All the elements of the locking loop are working at room temperature. A very similar approach, but using Schottky harmonic mixer instead of super-lattice one, was been published in 2015 by Danylov et al. [19] and Bulcha et al. [20]. In [20] it was phase locked a $2.5 \mathrm{THz}$ laser, and in [19] $2.3 \mathrm{THz}$ and $2.9 \mathrm{THz}$ lasers of power correspondingly. All the experiments in [17], [18], [19] and [20] can be reflected by very simple scheme shown in fig. 3 .

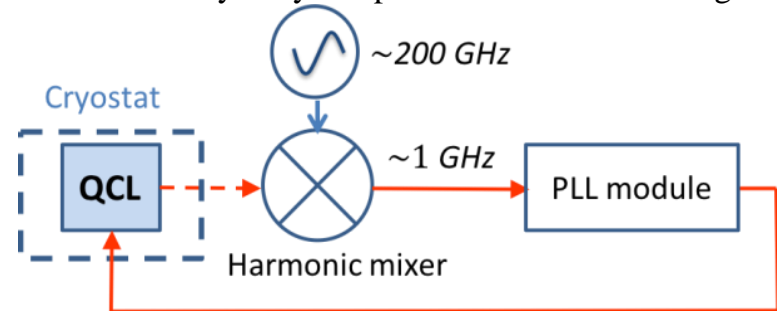

Fig. 3. Simplified block-diagram of QCL phase-locking shown in [17], [18], [19] and [20].

The latest conceptually unique method of QCL phase locking was presented in 2017 by Freeman et al. [21]. The $2 \mathrm{THz} 1.7 \mathrm{~mW}$ QCL was locked not by an active feed-back system but by injection locking method. Employing infrared frequency combs and InGaAs photomixers a reference signal of $100 \mathrm{nW}$ was generated at frequency very close to the QCL frequency. This signal was injected into the QCL and synchronized its radiation.

To summarize, the most promising are four techniques: [15]/[16]; [17]/[18] ; [19]/[20] and [21].

However, only in [18] it was shown phase locking at $4.7 \mathrm{THz}$, all the other QCLs are below $3 \mathrm{THz}$. Though, one of the most interesting applications for QCL is a role of $\mathrm{LO}$ in on-board heterodyne receiver at 4.7 $\mathrm{THz}$ to observe atomic oxygen line [4-8].

\section{References}

[1] R. Köhler, A. Tredicucci, et. al. // Terahertz semiconductor heterostructure laser // Nature 417, 156-159, 2002.

[2] A. W. M. Lee, Q. Qin, et al. // "Real-time terahertz imaging over a standoff distance ( $>25$ meters) // Appl. Phys. Lett. ,89, 141125, 2006.

[3] H.-W. Hübers, S. G. Pavlov, et. al. // High-resolution gas phase spectroscopy with a distributed feedback terahertz quantum cascade laser // Appl. Phys. Lett. 89, 0611152006. [4] H. Richter, M. Weinold, et al // 4.7-THz Local Oscillator for the GREAT Heterodyne Spectrometer on SOFIA
// IEEE Trans. on TST 5, 539-535, 2015.

[5] https://www.sron.nl/missions-astrophysics/gusto

[6] http://millimetron.ru/index.php/en/

[7] http://www.locussatellite.com/

[8] J. Fortney et al. https://arxiv.org/abs/1803.07730

[9] A. L. Betz, R.T. Boreiko, et al. // Frequency and phaselock control of a $3 \mathrm{THz}$ quantum cascade laser // Opt. Lett. 30, 1837-1839, 2005

[10] A. Baryshev, J. N. Hovenier, A. J. L. Adam, I. Kaalynas, J. R. Gao, T. O. Klaassen, B. S. Williams, S. Kumar, Q. Hu, and J. L. Reno // Phase locking and spectral linewidth of a two-mode terahertz quantum cascade laser // Appl. Phys. Lett. 89, 031115 , 2006.

[11] D. Rabanus, U. U. Graf, M. Philipp, O. Ricken, J. Stutzki, B. Vowinkel, M. C. Wiedner, C. Walther, M. Fischer, and J. Faist // Phase locking of a 1.5 Terahertz quantum cascade laser and use as a local oscillator in a heterodyne HEB receiver // Opt. Express 17, 1159-1168, 2009.

[12] P. Khosropanah, A. Baryshev, W. Zhang, W. Jellema, J. N. Hovenier, J. R. Gao, T. M. Klapwijk, D. G. Paveliev, B. S. Williams, S. Kumar, Q. Hu, J. L. Reno, B. Klein, and J. L. Hesler // Phase locking of a $2.7 \mathrm{THz}$ quantum cascade laser to a microwave reference // Opt. Lett. 34, 2958-2960 , 2009.

[13] D. G. Paveliev, Yu. I. Koschurinov, V. M. Ustinov, A. E. Zhukov, F. Lewen, C. Endres, A. M. Baryshev, P. Khosropanah, W. Zhang, K. F. Renk, B. I. Stahl, A. Semenov, and H.-W. Huebers // conf. proc., ISSTT, 319, 2008. [14] L. Consolino, A. Taschin, P. Bartolini, S. Bartalini, P. Cancio, A. Tredicucci, H. E. Beere, D. A. Ritchie, R. Torre, M. S. Vitiello, and P. De Natale // Phase-locking to a freespace terahertz comb for metrological-grade terahertz lasers // Nat. Commun. 3, 1040.

[15] S. Barbieri, P. Gellie, G. Santarelli, L. Ding, W. Maineult, C. Sirtori, R. Colombelli, H. Beere, and D. Ritchie // Phase-locking of a 2.7, THz quantum cascade laser to a mode-locked erbium-doped fibre laser // Nat. Photonics 4, 636-640, 2010.

[16] M. Ravaro, C. Manquest, C. Sirtori, S. Barbieri, G. Santarelli, K. Blary, J. F. Lampin, S. P. Khanna, and E. H. Linfield // Phase-locking of a $2.5 \mathrm{THz}$ quantum cascade laser to a frequency comb using a GaAs photomixer //", Opt. Lett., vol. 36, pp. 3969-3971, 2011.

[17] D. J. Hayton, A. Khudchenko, D. G. Pavelyev, J. N. Hovenier, A. Baryshev, J. R. Gao, T. Y. Kao, Q. Hu, J. L. Reno, and V. Vaks // Phase-locking of a 3.4-THz Quantum Cascade Laser using a harmonic super-lattice mixer // Appl. Phys. Lett. 103, 051115.

[18] A. Khudchenko, D. J. Hayton, D. G. Pavelyev, A. Baryshev, J. R. Gao, T. Y. Kao, Q. Hu, J. L. Reno, V. Vaks // Phase locking a $4.7 \mathrm{THz}$ Quantum Cascade Laser using a Super-lattice Diode as Harmonic Mixer // conf. proc. IRMMW-THz, 2014.

[19] A. Danilov, N. Erickson, A. Light, and J. Waldman, // Phase locking of 2.324 and 2.959 terahertz quantum cascade lasers using a Schottky diode harmonic mixer // Opt. Lett., vol. 40, pp. 5090-5092, 2015.

[20] B.T. Bulcha, J.L. Hesler, A. Valavanis, V. Drakinskiy, J. Stake, R. Dong, J.X. Zhu, P. Dean, L.H. Li, A.G. Davies, E.H. Linfield, N.S. Barke. // Phase locking of a $2.5 \mathrm{THz}$ Quantum Cascade Laser to a microwave reference using $\mathrm{THz}$ Schottky mixer // conf. proc., IRMMW-THz, 2015.

[21] J. R. Freeman, L. Ponnampalam, H. Shams, R. A. Mohandas, C. C. Renaud, P. Dean, L. Li, A. G. Davies, A. J. Seeds, and E. H. Linfield // Injection locking of a terahertz quantum cascade laser to a telecommunications wavelength frequency comb // Optica, Vol. 4, No.9, pp. 1059-1064, 201 